\title{
Association of NQO1 C609T (Pro187Ser) with risk of Oral Submucous Fibrosis in Eastern Indian population
}

\author{
Sanjit Mukherjee ${ }^{1, \#, *}$, Atul Katarkar ${ }^{1}$, Jay Gopal Ray² and Keya Chaudhuri ${ }^{1,}$ \\ ${ }^{1}$ CSIR-Indian Institute of Chemical Biology, Kolkata, India; ${ }^{2}$ Department of Oral Pathology, \\ Dr. R. Ahmed Dental College \& Hospital, Kolkata, India \\ \# Present address: Genetics Branch, National Cancer Institute, Bethesda, USA
}

*Address for correspondence:

Sanjit Mukherjee, $\mathrm{PhD}$

Genetics Branch

Center for Cancer Research

National Cancer Institute

Building 37, Room 6138

Bethesda, MD 20892-4265

Tel: +12407157409

Email: sanjit.mukherjee@nih.gov;

mukherjeesanjit@yahoo.com 


\begin{abstract}
Present study explores the role of NADPH quinone oxidoreductase 1 (NQO1) C609T (Pro187Ser) polymorphism in susceptibility to and its probable role in malignant potentiality of Oral submucous fibrosis (OSF) a debilitating disease caused mainly by chewing arecanut. About $18 \%$ of the patients were detected with minor TT allele $(\mathrm{Ser} / \mathrm{Ser}) \mathrm{p}=0.026$, while both CT (Pro/Ser) and TT (Ser/Ser) allele, $\mathrm{p}=0.003 \& 0.004$ respectively, was found to be higher in patients above 40yrs of age. NQO1 protein was $42 \%$ reduced in buccal tissues of heterozygous (Pro/Ser) carriers, whereas a 70\% reduction was observed in TT $(\mathrm{Ser} / \mathrm{Ser})$ patients. Decrease in NQO1 activity was noted in both in OSF tissues alone or when associated with malignancy.
\end{abstract}

Key words: Arecanut, Oral submucous fibrosis, NQO1, single nucleotide polymorphism, carcinogenesis.

\title{
Introduction
}

Upsurge in the popularity of commercially prepared areca nut preparations (popularly known as 'panmasala'), an increased uptake of this habit by young people easy access, effective price ranges and marketing strategies have contributed to a high prevalence of smokeless tobacco related oral carcinogenesis in India $(1,2)$. Chewing of tobacco with betel quid increases the concentrations of carcinogenic tobacco specific nitrosamines and reactive oxygen species in mouth (2) as an early sign of damage to oral mucosa, tobacco smokers and chewers often develop precancerous oral lesions such as leukoplakia and submucous fibrosis. Oral submucous fibrosis (OSF) is an insidious, chronic, progressive precancerous condition of the oral cavity and oropharynx with a high degree of malignant potentiality. A significant number of this precancerous condition converts into Oral Squamous Cell Carcinoma (OSCC), the rate being about 7.4-13\% (3). This disease is now a public health concern in many parts of the world including many southeast Asian countries, United Kingdom and South Africa though it is mainly prevalent in the Indian subcontinent in all age groups and across all socioeconomic strata.(4-7). The human $\mathrm{NAD}(\mathrm{P}) \mathrm{H}$ :quinone oxidoreductase 1 gene (NQO1; DT-diaphorase, Enzyme Commission (EC) number 1.6.99.2) occupies 17 kilobase pairs (kb) within a gene-rich region on chromosome 16 at 16q22.1 (8). This cytosolic flavoenzyme detoxifies quinones (a large class of aromatic compounds found commonly in plants, benzene metabolites, and chemotherapies) to hydroquinones or catechols. The enzyme $\mathrm{NAD}(\mathrm{P}) \mathrm{H}$ :quinone oxidoreductase 1 (NQO1) acts as an antioxidant by catalyzing a 2-electron reduction that bypasses the need for two 1-electron reductions that can result in the production of DNA and protein-damaging reactive oxygen species. In certain conditions (e.g., the presence of myeloperoxidase or autooxidants), NQO1 can contribute to the formation of reactive oxidation species via oxidative cycling and therefore can act as a prooxidant (9). NQO1 is constitutively expressed in most tissues including the bone marrow, where expression is thought to be highly inducible by xenobiotics with quinone moieties and is upregulated during times of oxidative or electrophilic stress.

The polymorphisms of NQO1gene have been characterized and known for about two decades (10). Of which a C-to-T substitution at position 609 of NQO1 cDNA, which codes for a prolineto-serine change at residue 187 is now well studied in most cancers. In cells with a TT Genotype NQO1 activity is not detected and lack of activity corresponds to a lack of NQO1 protein which undergoes rapid turnover via the ubiquitin mediated proteasomal degradation pathway (11). The 
prevalence of the TT genotype varies among ethnic groups (4-20\%), with the highest prevalence occurring in Asian populations $(12,13)$.

\section{Materials and Methods}

Patients and controls: A total of 179 patients with newly diagnosed cases of oral submucous fibrosis and 152 healthy control subjects having oral habit for a similar period were recruited from out patients department at Dr. R Ahmed Dental College \& hospital, Kolkata, India over a 3year period. All individuals enrolled in the study provided informed consent for their participation in the study. All participants completed a questionnaire providing information about their age, gender, ethnic origin, and use of processed arecanut products, smoking status or alcohol. Blood was collected from the antecubital vein, while biopsy sample was collected from the affected tissue after obtaining a written consent was obtained from all the patients after educating them about the importance and outcome of the study. All the study protocols were reviewed by an institutional ethical committee, and samples were collected by medical trained professionals. A part of the tissue was used for routine histopathological and immunohistochemical examination, while another part was used for further analysis of genetic or protein expression.

Polymorphism Genotyping: Genotyping was carried out by PCR RFLP analysis on DNA extracted from whole blood samples obtained from all participants in the study using primers forward: 5AAGCCCAGACCAACTTCT-3 ${ }^{\prime}$ and reverse:5 ATTTGAATTCGGGCGTCTGCTG-3 with an initial denaturation of $95^{\circ} \mathrm{C}$ for 2 mins followed by 35 cycles of $94^{\circ} \mathrm{C}$ for $30 \mathrm{secs}, 60^{\circ} \mathrm{C}$ for $30 \mathrm{secs}, 72^{\circ} \mathrm{C}$ for $1 \mathrm{~min}$ and a final elongation of $72^{\circ} \mathrm{C}$ for 10 mins. Subsequently, the PCR products were digested with $20 \mathrm{U}$ of HinfI (New England Biolabs, USA) for $3 \mathrm{hr}$ at $37^{\circ} \mathrm{C}$ and separated on a $6 \%$ polyacrylamide gel. The NQO1 wildtype allele shows a $172 \mathrm{bp}$ PCR product resistant to enzyme digestion, whereas the null allele shows a $131 \mathrm{bp}$ and a $41 \mathrm{bp}$ band. The frequency of the NQO1 genotypes was compared in the patient and control groups.

RNA isolation from oral mucosal tissue: Total RNA was extracted using Trizol reagent (Gibco BRL, Gaithersburg, MD) according to the manufacturer's protocol. Approximately $50 \mathrm{mg}$ of tissue was collected in $1 \mathrm{ml}$ Trizol reagent and homogenized using a handheld homogenizer. After the addition of $0.2 \mathrm{ml}$ chloroform, the mixture was vigorously shaken for $3 \mathrm{~min}$ at $22^{\circ} \mathrm{C}$ and centrifuged at $13000 \mathrm{rpm}$ for $15 \mathrm{~min}$ at $4^{\circ} \mathrm{C}$. An equal volume of isopropanol (chilled) was added and was kept in cold condition for $10 \mathrm{~min}$, RNA was precipitated by centrifugation at $13000 \mathrm{rpm}$ for $10 \mathrm{~min}$ at $4^{\circ} \mathrm{C}$. The pellet was washed twice with $70 \%$ ethanol, briefly dried under air, and dissolved in $100 \mu \mathrm{l}$ of diethylprocarbonate- treated water.

Quantitative realtime PCR of NQO1 mRNA: Isolated RNA from OSF and control tissues was immediately subjected to realtime RT PCR (using Takara PrimescriptTM one step realtime RT PCR kit) (Takara, Japan) analysis for detection of changes in expression of NQO1 mRNA according to the manufacturers protocol. Briefly, the PCR was carried out using following primers Forward 5'-GGG CAA GTC CAT CCC AAC TG-3' and Reverse 5'-GCAAGTCAG GGAAGCCTGGA-3' (230 bp) while for GAPDH the forward and reverse primers were 5'ATGGGGAAGGTGAAGGTCGG-3' and 5'-GGATGCTAAGCAGTTGGT-3' respectively, 
yielding a 470 bp product. The reaction mix contained 200 ng of RNA; PrimeScript One Step Enzyme Mix; 1X One Step Buffer; $20 \mu \mathrm{M}$ of each of the primers and RNase Free $\mathrm{dH} 2 \mathrm{O}$. The real-time RT-PCR cycling program involved an initial denaturation step at $95^{\circ} \mathrm{C}$ for 2 min, followed by 40 cycles of $15 \mathrm{~s}$ at $95^{\circ} \mathrm{C}$ and $30 \mathrm{~s}$ at $60^{\circ} \mathrm{C}$. Thermal cycling was performed in a BioRad iQ5 Real-Time PCR Detection System (Hercules, CA, USA).

Western blotting: The tissues were collected in PBS buffer containing protease inhibitor cocktail (Sigma-Aldrich, USA). Tissue extracts were prepared by lysis of PBS-washed cells in RIPA lysis buffer [150 mM NaCl 1\% Nonidet P-40 (vol/vol) 0.5\% AB-deoxycholate (vol/vol) $0.1 \%$ SDS ( vol/vol) 50mMTriszHCl (pH 8) $1 \mathrm{mM}$ DTT $1 \mathrm{mg} / \mathrm{ml}$ each of leupeptin, aprotinin, and pepstatin]. The insoluble pellet was discarded, and the protein concentration was determined by using Bradford reagent (Bio-Rad). Equal amounts of protein were mixed with sample buffer (4\% SDS $20 \%$ glycerol $10 \%$ 2-mercaptoethanol $0.125 \mathrm{M}$ TrisHCl), heated at $95^{\circ} \mathrm{C}$ for $5 \mathrm{~min}$, and loaded onto an $6 \%$ polyacrylamide- SDS gel and transferred onto a PVDF membrane (Millipore, Billerica MA). Primary antibodies used were - mouse monoclonal NQO1 (Cell Signalling Technology, Beverly, MA), After that suitable secondary antibody used was (goat anti rabbit or anti mouse) conjugated with ALP (1:2000 dilution) was added and incubated for 3 hours at room temperature followed by washing in TBST six times for ten minutes each to detect the protein levels.

Immunohistochemistry: Paraffin blocks each from wild type, heterozygous and homozygous mutant patients were selected for subsequent immunohistochemical investigations. Briefly, dissected oral tissues from biopsy specimen were paraffin embedded and $4-5 \mu \mathrm{m}$ thickness sections were collected on poly-L-lysine coated slides, after paraffin removal by xylene and rehydration, the slides were treated with citrate buffer for unmasking the antigen. The further immunostaining were performed using Novolink polymer detection system (Leica Microsystems, Switzerland). The endogenous peroxidase and protein were blocked using supplied blockers. The expression of NQO1 protein were detected using NQO1 monoclonal antibody (Ccell signaling technologies, Beverly, MA). After postprimary blocking the sections were incubated with novolink polymer and were then developed with DAB using supplied substrate buffer. The sections were then counterstained with haematoxylin and were observed under LEICA DM 3000 microscope ((Leica Microsystems, Switzerland).

NQO1 activity assay from affected and adjacent normal tissue: Oral biopsy tissues were obtained from patients (OSF and OSF associated with malignancy) both from the lesion and from the tissue adjacent to the lesion for measurements of NQO1 enzyme activity. The assay was performed as described by Ernster (1967) (14). and modified by Benson et al. (1980) (15) using DCPIP as a substrate. The reaction mix contained $25 \mathrm{mM}$ Tris $(\mathrm{pH} 7.4)$, with or without $0.07 \%$ bovine serum albumin (w/v) as activator, $200 \mu \mathrm{M}$ NADH, and $40 \mu \mathrm{M}$ DCPIP, and assays were carried out in different concentration of dicumarol. NQO1 activity is described as the dicumarol inhibitable decrease in absorbance at $600 \mathrm{~nm}$ with DCPIP as a substrate and is expressed in nanomoles of DCPIP reduced per minute per milligram of protein. Total protein in tissue and cell culture preparations was determined by the Biorad Protein assay kit (Hercules, CA, USA) using bovine serum albumin as a standard. 
Single cell gel electrophoresis: For single cell gel electrophoresis or 'COMET' assay, individuals were asked to rinse their mouths thoroughly for $2 \mathrm{~min}$ with tap water. The exfoliated buccal cells were collected from one or both cheeks from the controls, and in the area with lesion from the patients, depending on the region where the lesion was located, including cheek, soft and hard palate, dorsal, ventral and lateral surfaces of the oral cavity. Typically, sites with tough, leathery texture of mucosa, blanching of mucosa (persistent, white, marble-like appearance which may be localized, diffuse or reticular), quid-induced lesions (fine, white, wavy, parallel lines that do not overlap or criss-cross, are not elevated and radiate from a central erythematous area) were selected. Comet assay was performed and analyzed as described previously (16).

Statistical analysis: The calculation of genotypic and allele frequencies for cases and controls and association between polymorphisms of NQO1 C609T with the risk of oral submucous fibrosis among total and stratified population was estimated by computing Odds ratio (OR) and calculating 95\% Confidence Interval (CI) using a chi-square Table Analysis and Yates corrected $\mathrm{P}$ value was taken for significance. The expression of NQO1 protein was analyzed densitometricaly using Image $\mathbf{J}$ software after proper calibrations. The difference in protein expression was analyzed by Student " $t$ " test, $p$ value $<0.05$ was considered to be significant.

\section{Results:}

The total study population was stratified according to Gender (Male and Female), Median age (40 years), Inter-incisal distance (ID) as Grade I (ID>3cm), Grade II (ID 2-3cm), Grade III $(\mathrm{ID}<2 \mathrm{~cm}) \&$ Habit and are presented in Table 1 . We found that male $(65.9 \%)$ constituted a greater percentage of the study sample and that most of the OSF cases were in the age group of $>$ 40 years $(65.9 \%)$. When the total OSF cases were divided according to interincisal distance as grade I, II and III, maximum distribution was found for grade II (61.4\%).

Distribution of Genotypes of $\mathrm{NQO1}$ C609T polymorphism among the cases and controls: After PCR-RFLP analysis of the study samples (cases and control), the distribution of genotypes -Wild type $\mathrm{C} / \mathrm{C}$ allele, heterozygous $\mathrm{C} / \mathrm{T}$ allele and homozygous mutant $\mathrm{T} / \mathrm{T}$ alleles were observed in the stratified study population and different grades of OSF and are presented in Table 2 \& 3 respectively.

Overall the frequency of homozygous mutant type (18\%) was significantly higher than controls $(8.5 \%)$ [OR of 2.369 (1.167-4.804) $\mathrm{P}=0.026$ ]. When stratified according to age, both the heterozygous carrier and homozygous mutant variant was found to be higher in $>40$ aged OSF cases than in controls OR 4.5 (1.77-11.61) $\mathrm{P}=0.003$ and $\mathrm{OR} 6.4$ (1.92-21.35) $\mathrm{P}=0.004$ respectively. Higher prevalence of both the heterozygous and homozygous mutant trait was found among the grade I patients (52\% and 19\%). Interestingly, the patients who reported with well-formed carcinogenic lesions were mostly found to be homozygous carrier of the minor $\mathrm{T}$ allele.

NQO1 expression and epethilial DNA disintegration in different genotypes: An overall mRNA expression level was higher in patients with either genotype compared to controls indicating transcriptional up regulation of NQO1 in tissues (Figure 1A). In general a significantly decreased mean expression for NQO1 protein was observed in buccal mucosal tissues of patients having heterozygous $(42 \%)$ or homozygous $(70 \%)$ mutant conditions $(p=0.0055 \& p=0.0001$ 
respectively) indicating NQO1 degradation in homozygous / heterozygous mutant patients is a post-transcriptional event (Table 4) (Figure 1B).

Histochemical localization of NQO1 protein in oral epithelial cells (Figure 1C) is concurrent with western-blots and shows high concentrations of NQO1 expression in epithelium of the tissue sections obtained from OSF patients with wild type Pro/Pro genotype compared to almost focal expression in normal healthy epithelium. Sections show increased NQO1 expression around the basal cell layer in wild type trait. NQO1 expression in OSF patients with heterozygous trait showed faint localizations whereas patients with homozygous mutant trait showed almost no NQO1 expression in the basal cells or elsewhere.

Comparative DNA damage of OSF with other oral precancerous lesions from our previous study (16) and according to genotype in this study presents a general disintegrated DNA in patients with wild type genotype and this worsens with heterozygous or homozygous conditions (Figure 1D).

Lower NQO1 activity in OSF and OSF associated with malignancy tissues compared to their adjacent tissue: Figure 2A presents kinetics of NQO1-mediated reduction of DCPIP as a measure of NQO1 activity. The Vmax and Km of NQO1 at different concentration of dicoumarol inhibitor in Normal, OSF and OSF associated with malignancy patients are presented in supplementary table 1. With increase in concentration of Dicoumarol $(0,1.25,2.5,5$ and 10 $\mu \mathrm{M}$ ) there was a uniform decrease in Vmax of NQO1 but no significant change in Km value was detected either in presence or absence of the activator (BSA). This suggests there is a noncompetitive inhibition of the NQO1 activity in the presence of inhibitor. When compared to the OSF tissue alone there was about 2 fold decrease in activity $(\mathrm{p}=0.028)$ was noted with or without BSA (supplementary figure 1). This was about 10 fold decreased in OSF associated with malignant tissues $(\mathrm{p}<0.0001)$ thus explaining insignificant NQO1 activity.

\section{Discussion}

Oral submucous fibrosis is a high risk potentially malignant condition affecting any part of the oral cavity and pharynx, which could subsequently develop into oral cancer. The carcinogenic turnover of OSF may serve as a very important model to study various molecular changes that is taking place during oral carcinogenesis. In the present study we have tried to understand the pathogenesis and carcinogenesis of OSF in the light of NQO1 C609T polymorphism. In OSF mucosal tissues from patients carrying Ser/Ser genotype either traces or no NQO1 protein was detected, this was due to ubiquitin mediated proteosomal degradation. The prevalence of the Ser/Ser genotype varies among ethnic groups (4-20\%), with the highest prevalence occurring in Asian populations(12). In the present study, we observed a significantly higher number of subjects carrying the NQO1 homozygous mutant allele genotype in patients suffering from oral submucous fibrosis as compared to the control group. Mostly male patients with OSF reported to our outpatient department (OPD) compared to the female patients, and middle-aged/old patients (>40yrs) showed a greater association with the risk of the disease. This result is in line with previous investigations that showed an association between the NQO1 homozygous mutant genotype and other tumour types such as urothelial cancer, renal cancer, leukemias, lung cancer and cutaneous basal cell carcinoma (17-21). The mechanism underlying the correlation of NQO1 C609T polymorphism with the increased risk for developing various tumours likely resides in the different enzyme activities encoded by the NQO1 alleles. A case-control study of benzene 
exposed workers in China revealed increased risk of hematotoxicity in individuals with the T/T NQO1 genotype, suggesting that NQO1 protects against benzene toxicity.

The current study suggests that NQO1 C609T null allele acts in a recessive mode in the development of OSF, demonstrated by the fact that the heterozygous genotype frequency among OSF patients remains similar to that among the healthy controls. This indicates that in individuals with the NQO1 heterozygous genotype, the enzyme activity might be sufficient for protecting cells from damage by exogenous carcinogens important for the development of OSF. The determination of the NQO1 C609T genotype may be valuable as a stratification marker in future intervention trials for OSF and oral squamous cell carcinoma (OSCC). This finding may be particularly important in our country as most of the common people are habituated to areca chewing in different modes and are susceptible to development of OSF and eventually OSCC. Moreover since NQO1 C609T polymorphism was found to be positively associated with many solid as well as blood malignancies, therefore, a practical approach for cost-effective tumour screening may be designed taking other such polymorphisms into account. On the other hand, due to the relatively rare occurrence of the T/T genotype in the population, it is clear that in clinical practice NQO1 genotyping may be of importance only in combination with other risk factors. We also observed the effect of C609T polymorphism upon protein expression at the affected site of submucosal fibrosis in response to areca chewing which presented a $42 \%$ reduced expression of NQO1 protein in heterozygous patients, whereas a $70 \%$ reduced expression in homozygous Ser/Ser patients.

Presently oral cancers are increasing at an alarming rate and have an increasing mortality rate particularly in this part of the world, may serve as an model to study various interactions of proteins in carcinogenic turnover in the light of presence of different deleterious polymorphisms.

\section{References:}

1. Gupta PC, Sinor PN, Bhonsle RB, Pawar VS, \& Mehta HC (1998) Oral submucous fibrosis in India: a new epidemic? The National medical journal of India 11(3):113-116.

2. Nair UJ, Obe G, Friesen M, Goldberg MT, \& Bartsch H (1992) Role of lime in the generation of reactive oxygen species from betel-quid ingredients. Environmental health perspectives 98:203-205.

3. Aziz SR (1997) Oral submucous fibrosis: an unusual disease. Journal of the New Jersey Dental Association 68(2):17-19.

4. $\quad$ Seedat HA \& van Wyk CW (1988) Betel-nut chewing and submucous fibrosis in Durban. South African medical journal = Suid-Afrikaanse tydskrif vir geneeskunde 74(11):568571.

5. Maher R, Lee AJ, Warnakulasuriya KA, Lewis JA, \& Johnson NW (1994) Role of areca nut in the causation of oral submucous fibrosis: a case-control study in Pakistan. J Oral Pathol Med 23(2):65-69.

6. Zain RB, et al. (1997) A national epidemiological survey of oral mucosal lesions in Malaysia. Community dentistry and oral epidemiology 25(5):377-383.

7. Paul RR, et al. (2005) A novel wavelet neural network based pathological stage detection technique for an oral precancerous condition. Journal of clinical pathology 58(9):932938. 
8. Jaiswal AK, McBride OW, Adesnik M, \& Nebert DW (1988) Human dioxin-inducible cytosolic NAD(P)H:menadione oxidoreductase. cDNA sequence and localization of gene to chromosome 16. The Journal of biological chemistry 263(27):13572-13578.

9. Ross D, et al. (2000) NAD(P)H:quinone oxidoreductase 1 (NQO1): chemoprotection, bioactivation, gene regulation and genetic polymorphisms. Chemico-biological interactions 129(1-2):77-97.

10. Traver RD, et al. (1997) Characterization of a polymorphism in $\mathrm{NAD}(\mathrm{P}) \mathrm{H}$ : quinone oxidoreductase (DT-diaphorase). British journal of cancer 75(1):69-75.

11. Siegel D, et al. (2001) Rapid polyubiquitination and proteasomal degradation of a mutant form of NAD(P)H:quinone oxidoreductase 1. Molecular pharmacology 59(2):263-268.

12. Kelsey KT, et al. (1997) Ethnic variation in the prevalence of a common NAD(P)H quinone oxidoreductase polymorphism and its implications for anti-cancer chemotherapy. British journal of cancer 76(7):852-854.

13. Gaedigk A, et al. (1998) NAD(P)H:quinone oxidoreductase: polymorphisms and allele frequencies in Caucasian, Chinese and Canadian Native Indian and Inuit populations. Pharmacogenetics 8(4):305-313.

14. Ernster L \& Ronald W. Estabrook MEP (1967) [56] DT diaphorase. Methods in Enzymology, (Academic Press), Vol Volume 10, pp 309-317.

15. Benson AM, Hunkeler MJ, \& Talalay P (1980) Increase of NAD(P)H:quinone reductase by dietary antioxidants: possible role in protection against carcinogenesis and toxicity. pp 5216-5220.

16. Katarkar A, Mukherjee S, Khan MH, Ray JG, \& Chaudhuri K (2014) Comparative evaluation of genotoxicity by micronucleus assay in the buccal mucosa over comet assay in peripheral blood in oral precancer and cancer patients. Mutagenesis 29(5):325-334.

17. Wang YH, Lee YH, Tseng PT, Shen CH, \& Chiou HY (2008) Human NAD(P)H:quinone oxidoreductase 1 (NQO1) and sulfotransferase 1A1 (SULT1A1) polymorphisms and urothelial cancer risk in Taiwan. Journal of cancer research and clinical oncology 134(2):203-209.

18. Schulz WA, et al. (1997) Increased frequency of a null-allele for $\mathrm{NAD}(\mathrm{P}) \mathrm{H}$ : quinone oxidoreductase in patients with urological malignancies. Pharmacogenetics 7(3):235239.

19. Guha N, et al. (2008) NQO1 polymorphisms and de novo childhood leukemia: a HuGE review and meta-analysis. American journal of epidemiology 168(11):1221-1232.

20. Liu Y \& Zhang D (The NQO1 C609T polymorphism and risk of lung cancer: a metaanalysis. Asian Pac J Cancer Prev 12(11):3091-3095.

21. Clairmont A, et al. (1999) Association of NAD(P)H:quinone oxidoreductase (NQO1) null with numbers of basal cell carcinomas: use of a multivariate model to rank the relative importance of this polymorphism and those at other relevant loci. Carcinogenesis 20(7):1235-1240. 


\section{Figure Legends:}

Figure 1: Effect of various genotypes on expression patterns of NQO1 and buccal tissue architecture. A) Increased mRNA expression wrt GAPDH of NQO1 in patients compared to controls indicating transcriptional up regulation in affected tissues. B) Expression levels of NQO1 protein in different genotypic conditions in OSF patients. C) Immunohistochemical localization shows high basal level expression of NQO1 especially along the rete-ridges and submucosal cells lining epethilium in OSF patients with Pro/Pro allele (ii) compared to healthy subjects (i). Little or no detectable expression is observed in heterozygous (Pro/Ser) (iii) or homozygous (Ser/Ser) (iv) oral mucosal tissue. D) DNA damage as evident from 'COMET' assay performed on exfoliated buccal tissue from OSF patients with different genotypes. DNA disintegration worsens with heterozygous or homozygous mutant conditions.

Figure 2: NQO1 activity as measured by reduction of DCPIP in presence of dicoumarol with or without activator. The OSF with malignant lesion patients (OSF+MAL) carrying Ser/Ser allele presents reduced or no activity of NQO1 compared to normal or OSF patients with no malignancy. 
Table1: Demographic variables of the studied population

\begin{tabular}{lll}
\hline & OSF patients (179) & Healthy Controls (152) \\
\hline $\begin{array}{l}\text { Age } \\
\text { Range }\end{array}$ & $14-73$ & $18-78$ \\
Mean & 38 & 37 \\
Gender & & \\
Male & $118(65.9 \%)$ & $74(48.6 \%)$ \\
Female & $61(34.1 \%)$ & $78(52.4 \%)$ \\
Age above 40 yrs & $118(65.9 \%)$ & $94(61.8 \%)$ \\
Age below 40 yrs & $61(34.1 \%)$ & $58(39.1 \%)$ \\
OSF grade & & \\
Grade I $(>35 \mathrm{~mm})$ & $36(20.11 \%)$ & NA \\
Grade II $(20-35 \mathrm{~mm})$ & $110(61.4 \%)$ & NA \\
Grade III $(<19 \mathrm{~mm})$ & $33(18.43 \%)$ & NA \\
\hline
\end{tabular}


Table2: Genotypic distribution of NQO1 C 609 T polymorphism among cases and controls:

\begin{tabular}{llllll}
\hline \multicolumn{2}{c}{ Cases } & Normal & OR* & 95\% CI** & P Val \\
\hline $\begin{array}{lllll}\text { Total population } \\
\text { Pro/Pro }\end{array}$ & & & & & \\
Pro/Ser & $60(44.7)$ & $77(50.65)$ & ref & & \\
Ser/Ser & $32(17.8)$ & $13(8.5)$ & $2.369 *$ & $1.167-4.804$ & 0.026
\end{tabular}

\section{Below 40 yrs}

Pro/Pro $60(46.87) \quad 32(34.04) \quad$ ref

Pro/Ser $48(37.5)$ $53(56.3)$

0.493

$0.27-0.87$

0.024

Ser/Ser

$20(15.6) \quad 9(9.5)$

1.185

0.49-2.85

0.893

Above 40 yrs

Pro/Pro $20(39.2) \quad 43(76.7) \quad$ ref

Pro/Ser $19(37.2) \quad 9(16.07)$

$4.5^{*}$

1.77-11.61 0.003

Ser/Ser

$12(23.5) \quad 4(7.1)$

$6.4^{*}$

1.92-21.35 0.004

Male

Pro/Pro

$52(44.06) \quad 30(40.5) \quad$ ref

Pro/Ser

$46(38.9) \quad 38(51)$

0.698

0.376-1.297

0.329

Ser/Ser

$20(16.9) \quad 6(8.1)$

1.923

$0.711-5.59$

0.3

Female

Pro/Pro

$28(45.9)$

$32(41.02)$

ref

Pro/Ser

$21(34.4)$

39 (50)

0.615

$0.297-1.276$

0.265

Ser/Ser

12 (11.4) $7(8.9)$

1.95

$0.95-5.15$

0.322

\section{Grade 1}

Pro/Pro

$10(27.7)$

Pro/Ser

$19(52.7)$

Ser/Ser

7 (19.44)

Grade 2

Pro/Pro

53 (48.18)

Pro/Ser

38 (30.3)

Ser/Ser

19 (17.27)

Grade3

Pro/Pro

17 (51.5)

Pro/Ser

$10(30.3)$

Ser/Ser

6 (18.18) 
Table 3: Presence of NQO1 C609 T polymorphism among Grades of OSF patients

\begin{tabular}{llll}
\hline & Grade 1 & Grade 2 & Grade3 \\
\hline Pro/Pro & $10(27.7)$ & $53(48.18)$ & $17(51.5)$ \\
Pro/Ser & $19(52.7)$ & $38(30.3)$ & $10(30.3)$ \\
Ser/Ser & $7(19.44)$ & $19(17.27)$ & $6(18.18)$ \\
\hline
\end{tabular}


Table4: Effect of presence of NQO1 C $609 \mathrm{~T}$ polymorphism on protein expression in patients

\begin{tabular}{llll}
\hline Genotype & NQO1 & & P value \\
\hline Pro/Pro Vs Pro/Ser & Pro/Pro & Pro/Ser & 0.0055 \\
& $1.4875 \pm 0.8046$ & $0.8789 \pm 0.5969$ & \\
Pro/Pro Vs Ser/Ser & Pro/Pro & Ser/Ser & 0.0001 \\
& $1.4875 \pm 0.8046$ & $0.4297 \pm 0.2492$ & \\
\hline
\end{tabular}


Figure 1

A

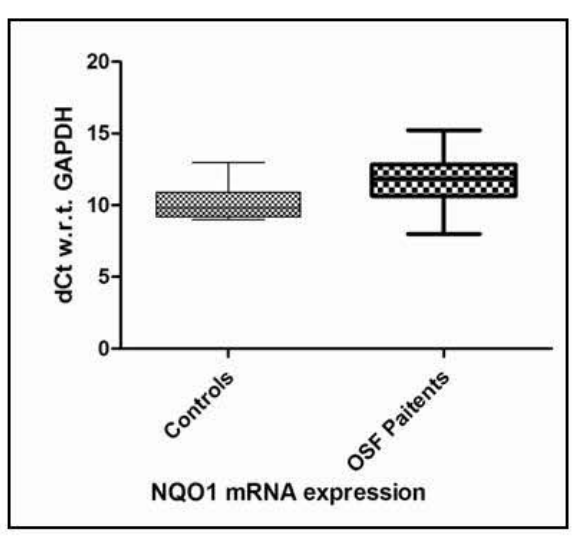

B

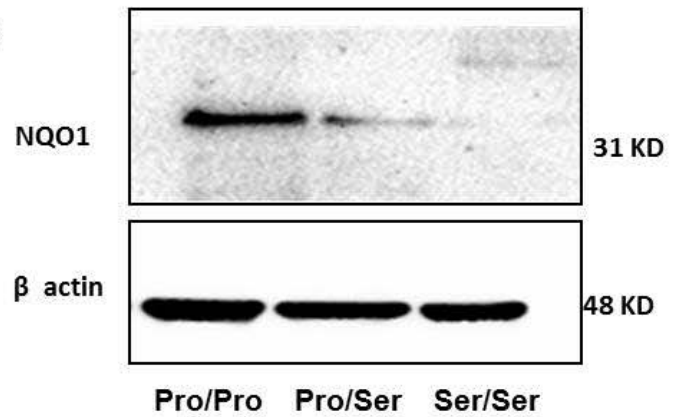

D

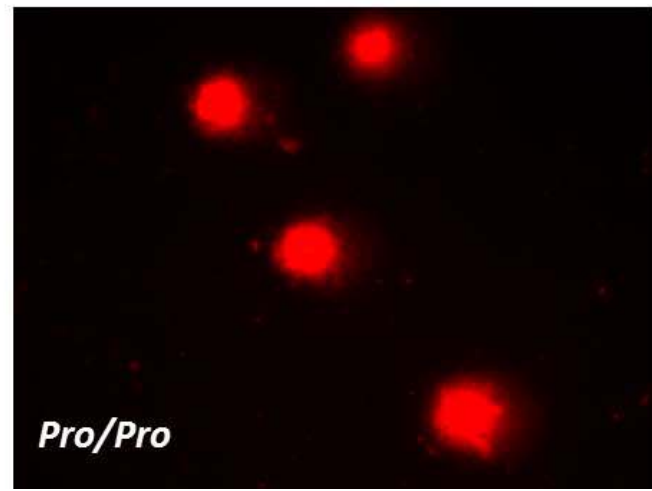

C
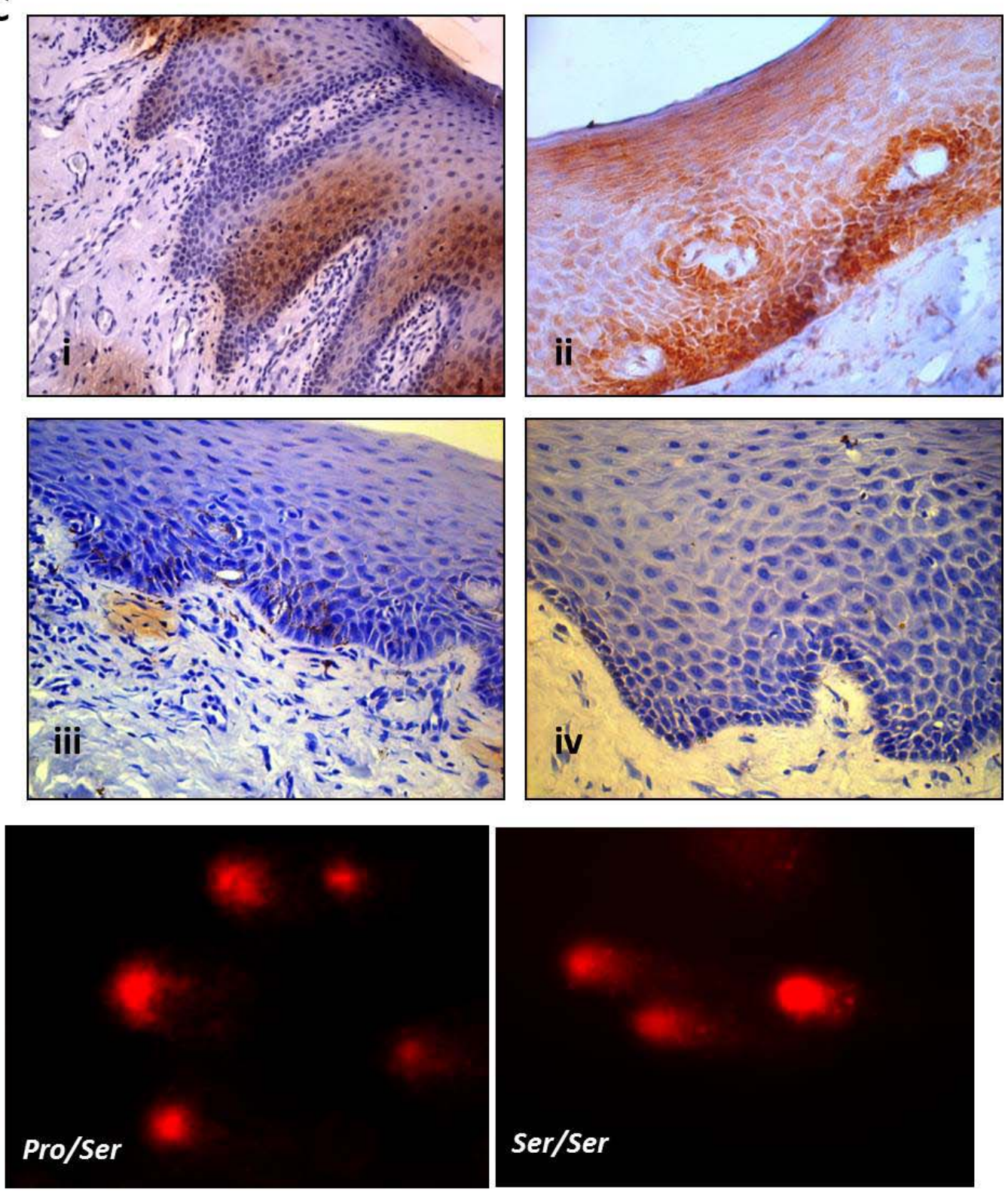

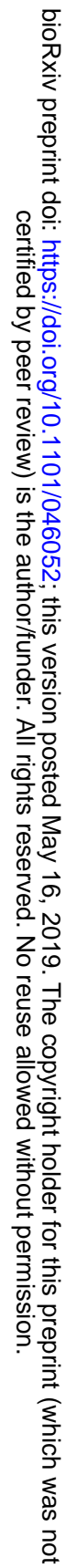


Figure 2

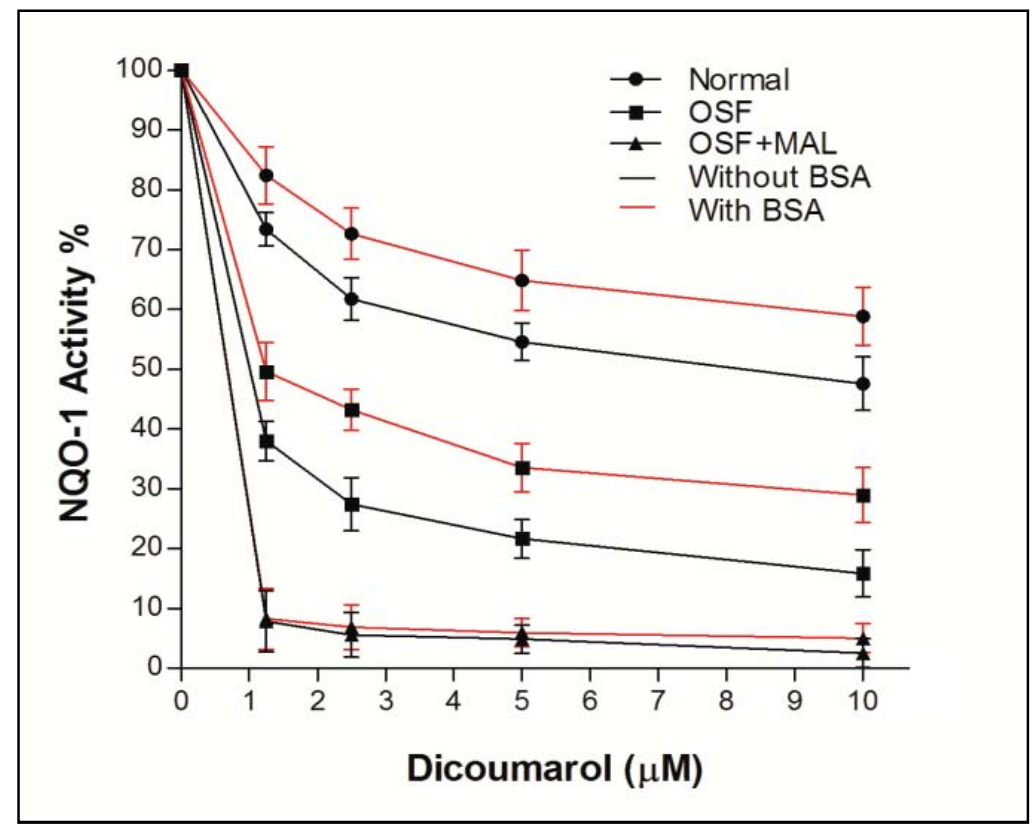

